\title{
Comparação de Metodologias de Predição de Valores Genéticos Utilizando Dados Simulados ${ }^{1}$
}

\author{
José Braccini Neto², Martinho de Almeida e Silva ${ }^{3,6}$, Ricardo Frederico Euclydes ${ }^{4}$, \\ Paulo Sávio Lopes ${ }^{4,6}$, Robledo de Almeida Torres ${ }^{4}, 6$, Adair José Regazzi ${ }^{5}, 6$
}

RESUMO - Foram simuladas, utilizando o programa GENESYS, sete populações com diferentes médias genéticas e estruturas de dados resultantes da substituição de $0,10,25,50,75,90$ e 100\% de reprodutores, com o objetivo de comparar a metodologia de modelos mistos (MMM) com a metodologia GenSys, por meio de suas acurácias e variâncias do erro de predição (PEV), estimadas com base no modelo de reprodutor e no modelo animal. No modelo de reprodutor, à medida que se aumentava a intensidade de seleção, ocorriam diminuição na acurácia e aumento da PEV de ambas as metodologias, em razão da menor acurácia na estimação dos níveis do efeito fixo e da diminuição do número médio de progênies/reprodutor. A superioridade da MMM no modelo de reprodutor foi atribuída à maior acurácia de estimação dos efeitos fixos. No modelo animal, as tendências foram diferentes. Na MMM, à medida que se aumentava a intensidade de seleção, a acurácia se elevava e a PEV permanecia praticamente constante, enquanto, na GenSys, ocorriam diminuição da acurácia e aumento da PEV. O aumento da acurácia, na MMM, decorreu do maior número de conexões genéticas, enquanto a diminuição, na GenSys, foi atribuída à menor acurácia de estimação dos níveis do efeito fixo. A superioridade da MMM foi decorrente da maior acurácia na estimação dos níveis do efeito fixo e do uso da matriz de parentesco completa, que inclui a população-base com indivíduos nãoaparentados, não-endogâmicos e não-selecionados. Conclui-se que a MMM foi superior à GenSys, quanto à acurácia e à PEV, para a estrutura de dados selecionados simulada, principalmente para o modelo animal.

Palavras-chave: GenSys, modelo animal, modelo de reprodutor, modelos mistos

\section{Comparison of Methodologies for Predicting Breeding Values Using Simulated Data}

ABSTRACT - Seven populations with different genetic means and different data structures resulting from replacing 0, 10, 25, 50, 75, 90 and $100 \%$ of sires simulated by GENESYS program were used to compare the mixed-model methodology (MMM) with the GenSys methodology, according to the accuracies and predicted error variances (PEV) of the breeding values, estimated under a sire or an animal model. Under the sire model, lower accuracy and greater PEV for both methodologies were observed as selection intensity increased, due to the reduced accuracy of estimation of the fixed effects and the reduced average number of progenies per sire. The superiority of MMM under the sire model was due to increase in the accuracy of the estimated fixed effects. Under the animal model, the tendencies were different. For the MMM, accuracy increased and PEV remained practically constant, as selection intensity increased, whereas for the GenSys methodology, reduced accuracy and an increase on the PEV as selection intensity increased were observed. Greater accuracies for MMM were due to the increased number of genetic ties, whereas the reduced accuracy for GenSys was due to the lower accuracy of the estimation of the fixed effects. The superiority of MMM was a result of the greater accuracy of the levels of fixed effect estimates and the use of the full relationship matrix, which included a base-population of unrelated, non-endogamic and non-selected individuals. The MMM was superior to GenSys according to the accuracy and PEV, for this structure of simulated selected data, mainly under the animal model.

Key Words: animal model, GenSys, mixed-model, sire model

\section{Introdução}

A maioria dos dados disponíveis aos melhoristas da espécie animal não apresenta os requerimentos de amostragem aleatória, ou seja, os dados são provenientes de experimentos de seleção ou de rebanhos que estão sob seleção (Henderson, 1973; Meyer \& Thompson, 1984; Gianola et al., 1988; Fries \& Schenkel, 1993).

Como conseqüência, as esperanças, as variâncias e as (co)variâncias entre as observações e os valores genéticos são alteradas (Henderson, 1982, 1985), o que resulta em estimativas e predições viesadas,

\footnotetext{
1 Parte da tese do primeiro autor apresentada à UFV para obtenção do título de Doutorado em Zootecnia - UFV. Bolsa CAPES.

${ }^{2}$ Professor do DZ, UFRGS, Porto Alegre, RS, CEP: 90001-970. E.mail: jose.braccini@ufrgs.br

3 Professor da Escola de Veterinária da UFMG, Belo Horizonte, MG, CEP: 30161-970. E.mail: martinho@vet.ufmg.br

${ }^{4}$ Professor do DZO, UFV, Viçosa - MG, CEP: 36571-000

${ }^{5}$ Professor do DPI, UFV, Viçosa - MG, CEP: 36571-000.

${ }^{6}$ Bolsista do CNPq
} 
sendo que alguns métodos são menos vulneráveis à seleção que outros (Henderson, 1975b).

Além disso, dados de campo apresentam número desigual de observações por subclasse, muitas subclasses vazias e problemas de conexidade. Em populações de bovinos de corte, ovinos e suínos, em que a inseminação artificial é pouco utilizada, rebanhos ou grupos de animais contemporâneos podem estar isolados geneticamente ou pobremente conectados. Assim, erros na seleção são, provavelmente, maiores (Kennedy \& Trus, 1993). A menor acurácia na predição de valores genéticos tem papel mais importante em rebanhos nos quais se utiliza inseminação artificial, pois um único indivíduo pode contribuir com muitos filhos para a próxima geração.

De modo geral, dados provenientes de populações submetidas à seleção são analisados, ignorando-se os efeitos da seleção (Schaeffer, 1987).

Entretanto, Brito (1992) e Brito \& Fries (1994) propuseram a metodologia GenSys para dados selecionados provenientes de condições reais e práticas da produção bovina de corte, na qual se considera a conexidade dos dados para fornecer estimadores não-viesados com propriedades robustas frente a observações extremas.

Henderson (1975b) sugeriu a comparação de metodologias por meio de simulação no caso em que os métodos analíticos envolviam manipulações matemáticas difíceis.

Objetivou-se, neste trabalho, comparar, quanto à acurácia e à variância do erro de predição, a metodologia de modelos mistos (MMM) e a metodologia GenSys, em situações de dados simulados com efeito de seleção.

\section{Material e Métodos}

Os dados deste trabalho foram simulados por meio do programa GENESYS (Euclydes, 1996).

Para simulação de uma variável resposta objetivando-se comparar as metodologias, foi definido, arbitrariamente, um genoma de 4.000 centimorgans com 14 pares de cromossomos autossômicos e um par de cromossomos sexuais contendo 500 genes de ação aditiva. As freqüências gênicas iniciais dos locos foram simuladas com base na distribuição uniforme, com média e variância 0,5 . Foram simulados dois alelos por loco, cujos efeitos apresentaram distribuição normal padrão.
Os efeitos de ambiente foram simulados conforme distribuição normal, com média zero e variância variável, de modo a permitir herdabilidade igual a 0,23 .

Primeiramente, foi simulada uma populaçãocontrole; posteriormente, $10,25,50,75,90$ ou $100 \%$ dos machos da $\mathrm{G}_{0}$ (população-base), com fenótipos inferiores, foram substituídos por animais mais jovens da primeira geração $\left(\mathrm{G}_{1}\right)$, que apresentaram maiores valores fenotípicos. Esses procedimentos foram repetidos até a décima primeira geração $\left(G_{11}\right)$, o que resultou em sete diferentes níveis de desbalanceamento, conexidade e progresso genético, de acordo com o diferencial de seleção paterno empregado.

Para a população-controle, considerando-se o modelo de reprodutor, a estrutura de dados foi balanceada, pois foram utilizados os mesmos reprodutores (20) em todos as gerações (12), tendo cada um deles apresentado o mesmo número de progênies (60), por geração. Essa estrutura de dados apresentou conexidade entre efeito de reprodutor e geração, pois cada reprodutor tinha progênie em cada geração, enquanto no caso de $100 \%$ de substituição, considerando-se o modelo fixo, a estrutura de dados foi desbalanceada, apresentando subclasses vazias para os reprodutores substituídos, e desconexa, pois cada reprodutor somente foi usado em uma geração. Para os outros casos, considerando-se o modelo misto, o desbalanceamento e a conexidade foram intermediários entre essas duas.

Simulou-se um efeito fixo de ambiente de mais uma unidade, a cada geração, para caracterizar um progresso ambiental além do genético, ou seja, foi acrescido o valor um ao valor fenotípico dos indivíduos da $\mathrm{G}_{0}$; o valor dois ao valor fenotípico dos indivíduos da $\mathrm{G}_{1}$; e assim sucessivamente, até a $\mathrm{G}_{11}$.

Comparou-se a MMM com a GenSys, utilizando-se o modelo animal (MA) e o modelo de reprodutor (MR), que são descritas a seguir:

\section{Metodologia de modelos mistos}

No modelo animal, utilizaram-se dados de teste de progênie, teste de desempenho individual e os valores fenotípicos dos pais.

A avaliação genética dos indivíduos foi feita com base no seguinte modelo:

$$
\mathrm{y}=\mathrm{X} \beta+\mathrm{Zu}+\mathrm{e}
$$

em que y é um vetor coluna com 13.620 observações; $\mathbf{X}$, uma matriz $(13.620 \times 12)$ conhecida, que relaciona 
os elementos de $\beta$ com os elementos de $\mathbf{y} ; \boldsymbol{\beta}$, um vetor coluna com 12 elementos desconhecidos, representando o efeito fixo de geração; $\mathbf{Z}$, uma matriz (13.620 x 13.620) conhecida, que relaciona os elementos de $\mathbf{u}$ com os elementos de $\mathbf{y}$; $\mathbf{u}$, um vetor coluna com 13.620 elementos desconhecidos assumidos aleatórios, representando os valores genéticos aditivos; e e, um vetor com 13.620 elementos também desconhecidos e aleatórios, que representam os resíduos associados às observações.

Admite-se que as variáveis aleatórias dos vetores $\mathbf{u}$ e e tenham o primeiro e o segundo momento dados por:

$$
E\left[\begin{array}{l}
\mathrm{u} \\
\mathrm{e}
\end{array}\right]=\left[\begin{array}{l}
0 \\
0
\end{array}\right] \text { e } \operatorname{Var}\left[\begin{array}{l}
\mathrm{u} \\
\mathrm{e}
\end{array}\right]=\left[\begin{array}{ll}
\mathrm{G} & 0 \\
0 & \mathrm{R}
\end{array}\right] .
$$

Como conseqüência, $\boldsymbol{E}(\mathbf{y})=\mathbf{X} \boldsymbol{\beta}$ e $\operatorname{Var}(\mathbf{y})=\mathbf{Z G Z}^{\prime}+\mathbf{R}$

Cada elemento de u e e tem média zero. A matriz de (co)variância dos elementos de $\mathbf{u}$ é $\mathrm{G}=\mathrm{A} \boldsymbol{\sigma}_{\mathrm{u}}^{2}$, em que A é uma matriz (13.620 x 13.620) não-singular do numerador da matriz de parentesco entre os indivíduos; e $\boldsymbol{\sigma}_{u}^{2}$, um escalar que representa a variância genética aditiva dos indivíduos não-aparentados e nãoendogâmicos da população-base. Os elementos de e não são correlacionados e têm variância comum $\boldsymbol{\sigma}_{\mathrm{e}}^{2}$, ou seja, a matriz de (co)variância dos elementos de $\mathbf{e}$ é $\mathrm{R}=\mathrm{I} \boldsymbol{\sigma}_{\mathrm{e}}^{2}$, em que I é uma matriz identidade de dimensão 13.620.

Para obtenção das equações de modelos mistos (EMM), admite-se que as variâncias genética aditiva e residual sejam conhecidas. Na prática, esses valores paramétricos são substituídos pelas suas respectivas estimativas. Mediante simulação, esses valores foram conhecidos e, então, utilizados.

A estimação dos efeitos fixos e a predição dos valores genéticos foram obtidas pela solução das EMM, apresentadas a seguir:

$$
\left[\begin{array}{cc}
X^{\prime} R^{-1} X & X^{\prime} R^{-1} Z \\
Z^{\prime} R^{-1} X & Z^{\prime} R^{-1} Z+G^{-1}
\end{array}\right]\left[\begin{array}{c}
\beta^{o} \\
\hat{u}
\end{array}\right]=\left[\begin{array}{c}
X^{\prime} R^{-1} y \\
Z^{\prime} R^{-1} y
\end{array}\right]
$$

Para obter $\beta^{\circ}$ e $\hat{u}$, empregou-se o programa computacional MTDFREML (Boldman et al., 1995).

$\mathrm{Na}$ análise dos dados, por meio do modelo de reprodutor, utilizaram-se somente os dados dos filhos (teste de progênie).

A avaliação genética dos reprodutores foi feita com base no seguinte modelo:

$$
\mathrm{y}=\mathrm{Xg}+\mathrm{Zs}+\mathrm{e},
$$

em que $\mathbf{y}$ é o vetor de observações; $\mathbf{X}$ e $\mathbf{Z}$, as matrizes de incidência para geração $(\mathbf{g})$ e reprodutor $\left(\mathrm{s} \sim\left(0, \mathrm{~A} \boldsymbol{\sigma}_{\mathrm{s}}^{2}\right)\right)$; e e, o vetor de resíduos aleatórios $\sim\left(0, \quad \mathrm{I} \boldsymbol{\sigma}_{\mathrm{e}}^{2}\right)$.

\section{Metodologia GenSys}

Utilizando-se o mesmo modelo matricial da MMM, admite-se que os elementos de $\mathbf{u}$ tenham média $\mathbf{u}$ e os de e, média zero. As matrizes de (co)variância dos elementos de u e e são, respectivamente, $\mathrm{G}=\mathrm{I} \boldsymbol{\sigma}_{\mathrm{u}}^{2}$ e $\mathrm{R}=\mathrm{I} \boldsymbol{\sigma}_{\mathrm{e}}^{2}$, em que I é uma matriz identidade de dimensão $n$.

A estimação dos efeitos fixos é obtida pela resolução do sistema de equações abaixo:

$$
\left[\begin{array}{cc}
X^{\prime} R^{-1} X & X^{\prime} R^{-1} Z \\
Z^{\prime} R^{-1} X & Z^{\prime} R^{-1} Z
\end{array}\right]\left[\begin{array}{l}
\hat{\beta} \\
\hat{u}
\end{array}\right]=\left[\begin{array}{c}
X^{\prime} R^{-1} y \\
Z^{\prime} R^{-1} y
\end{array}\right]
$$

As Diferenças Esperadas nas Progênies (DEPs) dos pais são estimadas por:

$$
\hat{\mu}=\left(Z^{\prime} R^{-1} Z+G^{-1}\right)^{-1} Z^{\prime} R^{-1}(y-X \hat{\beta}),
$$

que representa as equações de índice de seleção de Lush, em que os valores verdadeiros dos efeitos de $\boldsymbol{\beta}$ são substituídos pelas suas estimativas.

As DEPs das progênies são obtidas pela metade da soma das DEPs dos pais mais metade da segregação mendeliana.

Tabela 1 - Correlações de Spearman entre valores genéticos verdadeiros e valores genéticos preditos pela metodologia de modelos mistos (MMM) e pela metodologia GenSys, com base no modelo de reprodutor (MR) e no animal (MA)

Table 1 - Sperman correlation between true breeding values and predicted breeding values by mixed-model methodology (MMM) and the GenSys methodology, under a sire (SM) and an animal model (AM)

\begin{tabular}{lcccccc}
\hline & & \multicolumn{2}{c}{ MMM } & & \multicolumn{2}{c}{ GenSys } \\
\cline { 3 - 4 } \cline { 7 - 7 } Subst $^{1}(\%)$ & $\mathrm{n}^{2}$ & MR & MA & & MR & MA \\
Replac $^{1}$ & & $S M$ & $A M$ & & $S M$ & $A M$ \\
\hline 0 & 20 & 0,982 & 0,670 & & 0,979 & 0,488 \\
10 & 40 & 0,967 & 0,813 & & 0,828 & 0,466 \\
25 & 70 & 0,937 & 0,862 & & 0,618 & 0,311 \\
50 & 120 & 0,664 & 0,852 & & 0,598 & 0,307 \\
75 & 170 & 0,524 & 0,895 & & 0,435 & 0,245 \\
90 & 200 & 0,491 & 0,906 & & 0,382 & 0,231 \\
100 & 220 & 0,504 & 0,921 & & 0,372 & 0,223 \\
\hline
\end{tabular}

${ }_{1}^{1}$ Proporção de substituição dos reprodutores.

2 Número de reprodutores testados.

${ }^{1}$ Replacing rate of sires.

${ }^{2}$ Number of tested sires. 
Para estimar $\hat{\beta}$ e $\hat{\mathrm{u}}$, utilizou-se o software GenSys.

$\mathrm{Na}$ análise dos dados, por meio do modelo de reprodutor, foram usados somente os dados dos filhos.

A avaliação genética dos reprodutores foi feita com base no seguinte modelo:

$$
\mathrm{y}=\mathrm{Xg}+\mathrm{Zs}+\mathrm{e},
$$

em que $\mathbf{y}$ é vetor de observações; $\mathbf{X}$ e $\mathbf{Z}$ são matrizes de incidência para geração (g) e reprodutor $\left(s \sim\left(s, I \sigma_{s}^{2}\right)\right)$ e $\mathbf{e}$, vetor de resíduos aleatórios $\sim\left(0, \quad \boldsymbol{I}_{\mathrm{e}}^{2}\right)$.

A acurácia de ambas as metodologias foi avaliada pela correlação de Spearman entre os valores genéticos verdadeiros e os preditos.

A variância do erro de predição (PEV) foi calculada com base em sua definição teórica (Kennedy \& Trus, 1993): $\mathrm{PEV}=\operatorname{Var}(\hat{\mathrm{u}}-\mathrm{u})$.

Para o cálculo da PEV na MMM, com base no modelo de reprodutor, e na metodologia GenSys, com base em ambos os modelos, as DEPs foram multiplicadas por dois.

\section{Resultados e Discussão}

Na Tabela 1, são apresentadas as correlações de Spearman entre os valores genéticos verdadeiros e os valores genéticos preditos, utilizando-se o MR e o MA na MMM e na metodologia GenSys.

No MR, verificou-se tendência similar para ambas as metodologias. À medida que se aumentava a intensidade de seleção, a acurácia diminuía.

Essa relação foi, possivelmente, atribuída à menor acurácia de estimação dos níveis do efeito fixo, à medida que se aumentou o efeito da seleção. Em ambas as metodologias, houve superestimação dos níveis do efeito fixo, ou seja, ambas as metodologias não foram capazes, com base no MR, de separar efeitos ambiental e genético. Provavelmente, o aumento do desbalanceamento ou do número de subclasses vazias, a diminuição da conexidade e o uso da matriz de parentesco incompleta (não-uso da $\mathrm{G}=\mathrm{A} \boldsymbol{\sigma}_{\mathrm{u}}^{2}$ ) tenham interferido na estimação dos níveis do efeito fixo.

Segundo Sorensen \& Kennedy (1984), para a partição correta da tendência fenotípica, deve-se utilizar a matriz de parentesco completa, ou seja, incluir todos os animais envolvidos no processo de seleção, não levando em conta se eles tiveram ou não progênies. Caso essa condição não seja satisfeita, como é o caso do modelo de reprodutor, estimadores de efeitos fixos e de resposta à seleção serão viesados (Henderson, 1975a).

Com relação ao modelo de reprodutor, além do problema de estimação do efeito fixo, outro fator que influencia na acurácia é o número de progênies/ reprodutor; para $0,10,25,50,75,90$ e $100 \%$ de substituição, o número médio de progênies/reprodutor foi de $660 ; 330 ; 186,6 ; 110 ; 77,6 ; 66$ e 60 , respectivamente. Ojala et al. (1985) e Tosh \& Wilton (1994) observaram aumento na correlação entre o valor genético verdadeiro e o predito, à medida que se aumentava o número de progênies/reprodutor. A superioridade da MMM deveu-se à pequena superioridade na precisão de estimação dos efeitos fixos, em razão, possivelmente, do uso da matriz $\mathbf{A} \boldsymbol{\sigma}_{\mathrm{s}}^{2} \mathrm{em}$ vez de $\boldsymbol{I}_{\mathrm{s}}^{2}$, utilizada pela GenSys.

Para o modelo animal, os resultados foram opostos. $\mathrm{Na} \mathrm{MMM}$, à medida que se elevava a intensidade de seleção, aumentava-se a acurácia, enquanto, na GenSys, ocorria diminuição.

No caso da MMM, observou-se que não houve relação entre qualidade de estimação de efeito fixo e efeito de seleção; assim, o fator que deve estar atuando na acurácia é a matriz de parentesco. Isto é possível, pois, segundo Tosh \& Wilton(1994), conexões genéticas (um reprodutor aparentado incluído na matriz A é uma conexão genética) têm importante efeito na acurácia. Para 0, 10, 25, 50, 75, 90 e 100\% de substituição, os números de reprodutores foram, respectivamente, 20, 40, 70, 120, 170, 200 e 220 . À medida que se elevava o número de reprodutores, ocorria aumento do número de conexões genéticas e, conseqüentemente, da acurácia.

No caso da GenSys, observou-se menor precisão de estimação dos níveis do efeito fixo, semelhantemente ao MR.

A superioridade da $\mathrm{MMM}$ foi atribuída à melhor qualidade de estimação dos níveis do efeito fixo e ao uso da matriz de parentesco completa, que inclui a população-base com indivíduos não-aparentados, nãoendogâmicos e não-selecionados. Segundo Kennedy et al. (1988), pela matriz de parentesco, os valores genéticos dos animais são expressos como funções lineares de valores genéticos dos animais da populaçãobase e subseqüentes contribuições de amostragem Mendeliana, que não são influenciadas pela seleção e consideram a endogamia.

Ao comparar os modelos dentro de cada metodologia, observa-se que, na MMM, as tendências 
do MR e do MA foram opostas, cruzando próximo ao nível 25\% de seleção. A superioridade do MR em relação ao MA, nas menores proporções de substituição, decorreu possivelmente do maior número de progênies/reprodutor (Tosh \& Wilton, 1994). Apesar da maior acurácia na estimação dos efeitos fixos do MA, nas menores proporções de substituição, o uso das (co)variâncias entre os indivíduos é que determina sua superioridade após o nível $25 \%$ de seleção. Para a GenSys, as tendências do MR e do MA foram semelhantes, sendo a superioridade do MR, em relação ao MA, atribuída, possivelmente, à maior acurácia do teste de progênie, para a mesma qualidade de estimação de efeito fixo em ambos os modelos e para a mesma quantidade de informação.

$\mathrm{Na}$ Tabela 2, constam as variâncias dos erros de predição com base no MR e no MA, para ambas as metodologias.

Para o modelo de reprodutor, houve tendência semelhante em ambas as metodologias, ou seja, como o aumento da intensidade de seleção, ocorreu incremento da PEV com mais intensidade na metodologia GenSys, em decorrência da menor qualidade de estimação dos níveis do efeito fixo e da redução do número de progênies/reprodutor, à medida

Tabela 2 - Variâncias do erro de predição (PEV), estimadas pela metodologia de modelos mistos (MMM) e pela metodologia GenSys, com base no modelo de reprodutor (MR) e no modelo animal (MA)

Table 2 - Predicted error variances (PEV) estimated by mixed-model methodology (MMM) and the GenSys methodology, under a sire (SM) and an animal model (AM)

\begin{tabular}{lrrrrrr}
\hline & & \multicolumn{2}{c}{ MMM } & & \multicolumn{2}{c}{ GenSys } \\
\cline { 3 - 4 } Subst $^{1}(\%)$ & $\mathrm{n}^{2}$ & MR & MA & & MR & MA \\
Replac $^{1}$ & & \multicolumn{1}{c}{$S M$} & \multicolumn{1}{c}{$A M$} & & \multicolumn{1}{c}{$S M$} & $A M$ \\
\hline 0 & 20 & 4,037 & 87,695 & & 3,181 & 125,527 \\
10 & 40 & 15,396 & 91,179 & & 72,647 & 209,537 \\
25 & 70 & 67,332 & 92,984 & & 218,107 & 316,718 \\
50 & 120 & 160,327 & 103,409 & & 190,800 & 312,089 \\
75 & 170 & 265,335 & 98,557 & & 313,353 & 442,997 \\
90 & 200 & 317,163 & 99,487 & & 375,931 & 495,090 \\
100 & 220 & 368,543 & 98,715 & & 434,133 & 573,113 \\
\hline
\end{tabular}

1 Proporção de substituição dos reprodutores.

2 Número de reprodutores testados.

${ }_{1}^{1}$ Replacing rate of sires.

2 Number of tested sires.

R. Bras. Zootec., v.33, n.6, p.1683-1688, 2004 (Supl. 1) que se aumentou o efeito da seleção. A superioridade da MMM foi atribuída à maior precisão de estimação dos efeitos fixos.

Para a MMM sob modelo animal, à medida que se aumentou a intensidade de seleção, a PEV permaneceu praticamente constante, enquanto, na metodologia GenSys, ocorreu aumento da PEV.

No caso da MMM, o maior número de conexões genéticas não interferiu na $\mathrm{PEV}$. Tosh \& Wilton (1994) encontraram correlação de $-0,10$ entre conexões genéticas e PEV. Esses autores afirmaram que a diminuição na $\mathrm{PEV}$, por meio do uso de animais aparentados, depende de muitos fatores, como acurácia do registro do teste de desempenho individual, número e coeficientes de parentesco e acurácia da avaliação dos parentes.

Para a metodologia GenSys, constatou-se menor precisão de estimação dos níveis do efeito fixo, à semelhança do que ocorreu com o MR.

Assim como na correlação, a superioridade da MMM foi atribuída à melhor qualidade de estimação dos níveis do efeito fixo e ao uso da matriz de parentesco completa.

Quando se compararam os modelos dentro de cada metodologia, observou-se que, na MMM, houve aumento da PEV para o MR, enquanto o MA permaneceu constante, à medida que se elevou a intensidade de seleção. O ponto de interseção das curvas ocorreu logo após o nível de $25 \%$ de substituição. Na metodologia GenSys, houve incremento na PEV, quando aumentou a intensidade, em ambos os modelos, reprodutor e animal. Na MMM, a superioridade do $\mathrm{MR}$ em relação ao $\mathrm{MA}$, nas menores proporções de substituição, decorreu possivelmente do maior número de progênies/reprodutor, apesar de sua menor acurácia na estimação dos efeitos fixos. $\mathrm{Na}$ metodologia GenSys, a superioridade do MR, em relação ao MA, foi possivelmente atribuída à maior acurácia do teste de progênie em relação ao teste de performance para mesma qualidade de estimação de efeito fixo, em ambos os modelos, e para a mesma quantidade de informação.

\section{Conclusões}

A metodologia de modelos mistos foi superior à GenSys, quanto à acurácia e à variância do erro de predição, para a estrutura de dados selecionados simulada, principalmente para o modelo animal. 


\section{Agradecimento}

Ao Prof. Luiz Alberto Fries, por sua gentileza em nos ceder o software GenSys.

\section{Literatura Citada}

BOLDMAN, K.G.; KRIESE, L.A.; Van VLECK, L.D. et al. A manual for use of MTDFREML. A set of programs to obtain estimates of variances and covariances [DRAFT] Lincoln: USDA/ARS, 1995. 114p.

BRITO, F.V.; FRIES, L.A. Proposta de um método para avaliação genética de bovinos de corte. Revista Brasileira de Zootecnia, v.23, n.2, p.181-188, 1994.

BRITO, F.V. Comparações entre metodologias para avaliação genética de bovinos de corte. Porto Alegre: Universidade Federal do Rio Grande do Sul, 1992. 177p. Dissertação (Mestrado em Zootecnia) - Universidade Federal do Rio Grande do Sul, 1992.

FRIES, L.A.; SCHENKEL, F.S. Estimation and predtiction under a selection model. In: SIMPÓSIO SOBRE PRODUÇÃO E CLASSIFICAÇÃO DE CARCAÇAS, 30., 1993, Rio de Janeiro. Anais... Rio de Janeiro: Sociedade Brasileira de Zootecnia, 1993. p.1-22.

GIANOLA, D.; IM, S.; FERNANDO, R.L. Prediction of breeding value under Henderson's selection model: a revisitation. Journal of Dairy Science, v.71, n.10, p.2790-2798, 1988.

HENDERSON, C.R. Best linear unbiased estimation and prediction under a selection model. Biometrics, v.31, n.2, p.423-447, 1975a.

HENDERSON, C.R. Best linear unbiased prediction in populations that have undergone selection. In: WORLD CONGRESS ON SHEEP AND BEEF CATTLE BREEDING, 1:Technical, 1980, Palmerston North. Proceedings... Palmerston North: The Dunmore Press Limited, 1982. v.2, p.191-200.

HENDERSON, C.R. Best linear unbiased prediction using relationship matrices derived from selected base populations. Journal of Dairy Science, v.68, n.2, p.443-448, 1985.
HENDERSON, C.R. Comparison of alternative sire evaluation methods. Journal of Animal Science, v.41, n.3, p.760-770, 1975b.

HENDERSON, C.R. Sire evaluation and genetic trends. In: ANIMAL BREEDING AND GENETICS SYMPOSIUM IN HONOR OF DR. JAY L. LUSH, 1972, Blacksburg. Proceedings... Champaign: ASAS/ADSA, 1973. p.10-41.

KENNEDY, B.W.; SCHAEFFER, L.R.; SORENSEN, D.A. Genetic properties of animal models. Journal of Dairy Science, v.71, p.17-26, 1988. Supl.2.

KENNEDY, B.W.; TRUS D. Considerations on genetic connectedness between management units under na animal model. Journal of Animal Science, v.71, p.2341-2352, 1993.

MEYER, K.; THOMPSON, R. Bias in variance and covariance component estimators due to selection on a correlated trait. Journal of Animal Breeding and Genetics, v.101, p.33-50, 1984

OJALA, M.; SYVÄJÄRVI, J., HELLMAN, T. Effect of the size of the class of comparison on the accuracy of a sire's evaluation. Journal of Animal Breeding and Genetics, v.102, p.91-94, 1985.

SCHAEFFER, L.R. Estimation of variance components under a selection model. Journal of Dairy Science, v.70, n.3, p.661-671, 1987.

SORENSEN, D.A.; KENNEDY, B.W. Estimation of response to selection using least squares and mixed model methodology. Journal of Animal Science, v.58, n.5, p.1097-1106, 1984.

TOSH, J.J., WILTON, J.W. Effects of data structure on variance of prediction error and accuracy of genetic evaluation. Journal of Animal Science, v.72, n.10, p.2568-2577, 1994. 